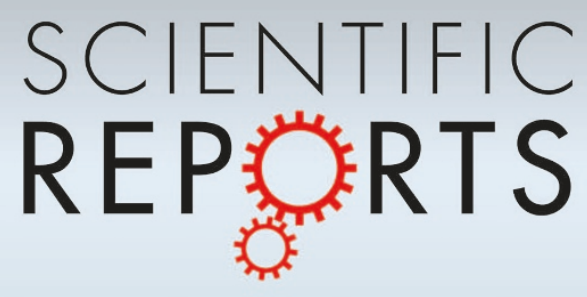

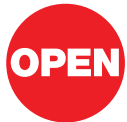

SUBJECT AREAS: IMMUNE TOLERANCE

CLONAL SELECTION ANTIGEN-PRESENTING CELLS

LYMPHOCYTES

Received

1 March 2013

Accepted

5 April 2013

Published

24 April 2013

Correspondence and requests for materials should be addressed to H.S. (sakano@mail. ecc.u-tokyo.ac.jp)

\section{A major allogenic leukocyte antigen in the agnathan hagfish}

\author{
Hiroyuki Takaba' ', Takeshi Imai ${ }^{1,2,3}$, Shoji Miki', Yasuyuki Morishita' ${ }^{4}$, Akihiro Miyashita', \\ Naoko Ishikawa ${ }^{5}$, Hirofumi Nishizumi' \& Hitoshi Sakano'
}

'Department of Biophysics and Biochemistry, Graduate School of Science, The University of Tokyo, Tokyo 113-0032, Japan, ${ }^{2}$ PRESTO, Japan Science and Technology Agency, Saitama 332-0012, Japan, ${ }^{3}$ Laboratory for Sensory Circuit Formation, RIKEN Center for Developmental Biology, Kobe 650-0047, Japan, ${ }^{4}$ Department of Molecular Pathology, Graduate School of Medicine,

${ }^{5}$ Department of Biological Sciences, Graduate School of Science, The University of Tokyo, Tokyo 1 13-0033, Japan.

All vertebrates, from jawless fish to mammals, possess adaptive immune systems that can detect and inactivate non-self-antigens through a vast repertoire of antigen receptors. Unlike jawed vertebrates, the hagfish utilizes variable lymphocyte receptors (VLRs) that are unrelated to immunoglobulin molecules but are diversified by copy-choice gene conversion mechanism. Here, we report that hagfish VLRs react with allogenic leukocyte antigens but not with self-antigens. We found that a highly polymorphic membrane protein, NICIR3, is recognized by VLRs as an allogenic leukocyte antigen (ALA). In a serological cross-reactivity test, a close correlation was observed between the amino acid differences in the protein sequences and the VLR cross-reactivities. This leukocyte antigen was predominantly expressed in phagocytic leukocytes, where it was associated with phagocytosed protein antigens. These findings suggest that a polymorphic leukocyte antigen, NICIR3/ALA, plays a pivotal role in jawless vertebrate adaptive immunity.

awless vertebrates possess acquired immunity but do not have immunoglobulin (Ig)-type antigen receptors that are diversified by $\mathrm{V}(\mathrm{D}) \mathrm{J}$-type recombination ${ }^{1-8}$. Variable lymphocyte receptors (VLRs), which consist of multiple leucine-rich repeat modules, were first identified in the sea lamprey by Pancer et al. in $2004^{9}$. The VLRs are structurally similar to the Toll-like receptors (TLRs) ${ }^{9-12}$ that recognize specific pathogens and its components ${ }^{13-15}$. A functional VLR gene is generated by the assembly of multiple variable gene segments known as a copy-choice gene conversion mechanism ${ }^{16,17}$. Thus, the evolutionary origin of adaptive immunity in jawless fish appears to be distinct from that of jawed vertebrates, in which the diversification process was acquired by an accidental insertion of transposons into the primordial $\operatorname{Ig}$ genes ${ }^{18-20}$. Although jawless and jawed vertebrates possess distinct adaptive immune systems using different strategies for gene rearrangement, both have to eliminate self-reactive lymphocytes to establish immunological tolerance, as somatic diversification of antigen receptors occurs in a random fashion and in an antigen-independent manner producing potentially hazardous specie $^{21-24}$. In this study, we investigate whether negative selection also takes place in the jawless fish VLRs. Furthermore, we studied a highly polymorphic leukocyte antigen, NICIR3/ALA, which may be involved in the recognition of phagocytosed protein antigens.

\section{Results}

Serum VLRs recognize allogenic leukocytes. Three distinct types of VLRs are known in the sea lamprey; VLR-A, VLR-B and VLR-C. Each is expressed in three separate lymphoid cell subpopulations in a mutually exclusive and monoallelic manner ${ }^{16,25,26}$. VLR-B molecules are secreted in blood and react to exogenous antigens, whereas VLRAs are expressed only in membrane-bound forms and VLR-Cs are not well-known ${ }^{13,25,26}$. Unlike sea lamprey, hagfish possess only two types of VLRs: VLR-A and VLR-B ${ }^{27,28}$. To determine the feature of the VLR-As and VLRBs of the hagfish, Eptatretus burgeri, polyclonal and monoclonal antibodies were generated against them (supplementary Fig. 1A-D). Immunoblot analyses detected VLR-Bs, but not VLR-As, in the serum (supplementary Fig. 1C). Thus, only VLR-B is secreted.

During the course of specificity analysis, we noticed that our monoclonal antibody (mAb 25D-9C) recognized serum VLR-B but not VLR-B in the paraformaldehyde-fixed leukocytes (Fig. 1A and B). Because mAb 25D-9C recognizes VLR-B leukocytes after antigen retrieval treatment $\left(121^{\circ} \mathrm{C}\right.$ for $\left.20 \mathrm{~min}\right)$, fixation may have masked the 
A

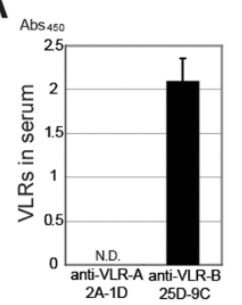

B
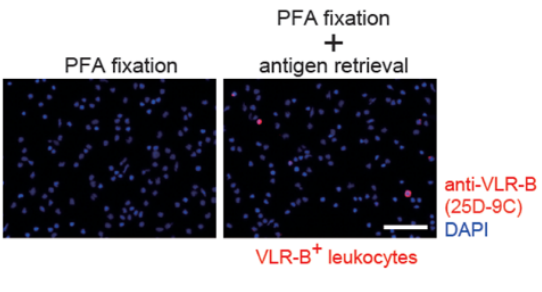

C

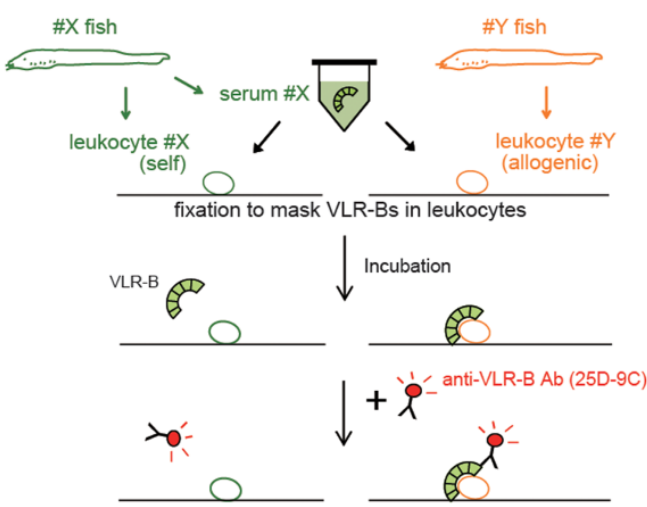

D

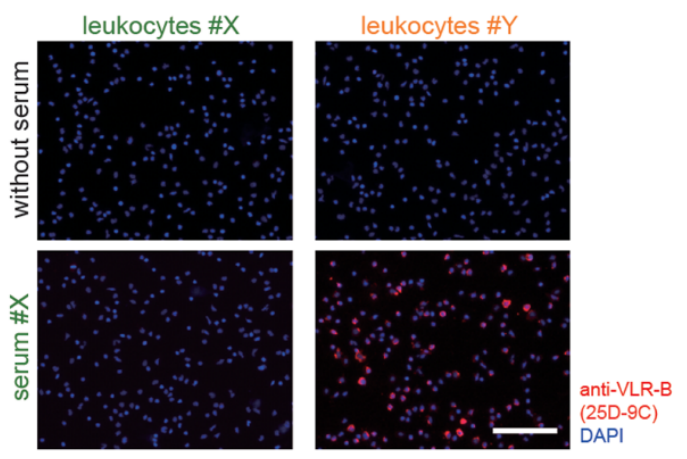

Figure 1 Secreted VLR-Bs react with allogenic leukocytes but not with self-ones. (A) ELISA of hagfish serum with mouse anti-VLR-A (2A-1G) and anti-VLR-B (25D-9C) monoclonal antibodies (mAb). Error bars indicate the s.e.m. ( $\mathrm{n}=12$ fish). These results show that hagfish VLR-Bs are secreted in blood. N.D. indicates less than 0.1. (B) Staining of VLR-B producing leukocytes. Buffy coat leukocytes from a hagfish were fixed on glass slides, masking VLR-Bs. Anti-VLR-B monoclonal antibodies (25D9C) recognized VLR-B producing leukocytes only after antigen retrieval treatment (heating at $121^{\circ} \mathrm{C}$ for $20 \mathrm{~min}$ at $100 \mathrm{kPa}$ in $0.01 \mathrm{M}$ citric acid, $\mathrm{pH}$ 6). A scale bar is $100 \mu \mathrm{m}$. (C) Schematic diagram of the serological assay. Buffy coat leukocytes and serum containing VLR-Bs were separately collected from the hagfish Eptatretus burgeri. Buffy coat leukocytes from fish \#X and fish \#Y were fixed on glass slides and treated with complementinactivated serum from fish \#X. Binding of serum-derived VLR-Bs was detected with mouse monoclonal antibodies (25D-9C) against secreted VLR-Bs (bottom). (D) Leukocyte staining with VLR-Bs. Anti-VLR-B monoclonal antibodies (25D-9C) do not recognize VLR-B producing leukocytes after fixation (top panels). As a result, only secreted VLR-Bs from the serum is detected with this antibody. Secreted VLR-Bs react with buffy coat leukocytes from different individuals (bottom right, red), but not with those isolated from the same individual (bottom left), suggesting allogenic recognition of leukocyte antigens. A scale bar is $100 \mu \mathrm{m}$.

epitope for this antibody. Taking advantage of this observation, we examined the alloreactivity of VLR-Bs using serological assay described below. In the human, natural antibodies (IgM and IgGs) are known to react to allogenic, but not self antigens on the hematocytes, e.g., erythrocytes and leukocyte ${ }^{29,30}$. However, it has never been tested whether the agnathan VLRs recognize allogenic antigens.

Leukocytes of buffy coat fraction ${ }^{9,27,28}$ and serum samples were isolated from the peripheral blood of different hagfish. Leukocytes were then fixed on glass slides and treated with the complementinactivated serum from self or non-self hagfish. We used anti-VLR-B monoclonal antibody mAb 25D-9C to detect the "serum-derived" VLR-Bs (Fig. 1A). It should be noted that mAb 25D-9C does not recognize VLR-Bs in fixed leukocytes (Fig. 1B). We found that VLRBs in the serum reacted to leukocytes from allogenic hagfish but not to autologous cells (Fig. 1C and D and supplementary Fig. 1E). VLRBs did not react to allogenic erythrocytes. These results indicate that VLR-Bs are negatively selected for self-leukocyte antigens in the hagfish, as has been known for immunoglobulins in jawed vertebrates $^{22}$.

We then examined the types of molecules recognized by VLR-Bs as allogenic antigens. We first hypothesized that these molecules would be carbohydrates, as in the case of mammalian blood antigens $^{29}$. However, VLR-B reactivity was not affected by glycosidase treatment. Because VLR-B reactivities varied among individuals, we assumed that this allogenic leukocyte antigen may be a polymorphic membrane protein.

A highly polymorphic leukocyte antigen. To identify such a polymorphic leukocyte antigen, we performed microarray analyses using mRNAs isolated from buffy coat leukocytes and erythrocytes, and searched for transmembrane proteins among the $10 \%$ most highly expressed proteins in leukocytes (but not in erythrocytes) as the initial criterion. A custom microarray was designed with approximately 24,000 hagfish leukocyte EST sequences ${ }^{31}$. After sequence analysis of 40 candidate genes, we found that a gene for immunoreceptor tyrosine-based activation motif-containing Ig superfamily receptor 3 (NICIR3) $)^{32}$ is the only polymorphic gene. NICIR3 was previously reported as a polymorphic transmembrane receptor with unknown functions by Haruta et al. ${ }^{32}$. Although the hagfish has three NICIR family genes, our analysis of the NICIR sequences from 25 fish samples confirmed that only NICIR3 is highly polymorphic (Fig. 2A and supplementary Fig. 2A). The extracellular portion of NICIR3 consists of two Ig domains ( $\mathrm{V}$ and $\mathrm{C}$ ), and the variable residues are clustered in the extracellular region (Fig. 2B and C).

NICIR3 is a major allogenic leukocyte antigen for VLR-Bs. To examine the alloreactivities of NICIR3 proteins and serum samples, we prepared buffy coat leukocytes and sera (secreted VLR-Bs) from eight hagfish specimens and performed serological tests for all combinations of leukocytes and serum samples. Each serum sample demonstrated variable reactivities to leukocytes from different hagfish (Fig. 3A). To identify the NICIR3 haplotypes, the coding sequences of two alleles (paternal and maternal) were amplified by PCR and were sequenced for all eight specimens. The differences in the deduced amino acid sequences were analyzed for alloreactivity. For each serum sample (\#X), the total residue differences with another individual (\#Y) were counted for both the maternal and paternal alleles. For example, in the combination of serum from \#X and buffy coat leukocyte from \#Y, where $\# \mathrm{X}$ has two allelic sequences, $\mathrm{AAAAA}$ and $\mathrm{AABAB}$, and $\# \mathrm{Y}$ has $\mathrm{ABABA}$ and $\mathrm{BBBCC}$, the difference (the expected antigenicity sore) would be counted as $1+2+0+2+1=6$. For the combination of serum \#Y and buffy coat leukocyte \#X, the difference would be $0+2+0+2+1=5$. In this way, expected antigenicity scores were calculated for 64 different combinations of serum and buffy coat leukocyte samples isolated from eight different individuals (Fig. 3B). In Fig. 3C, the calculated amino acid differences among the different haplotypes are plotted against the observed alloreactivities (VLR-B binding). A positive correlation was 
A

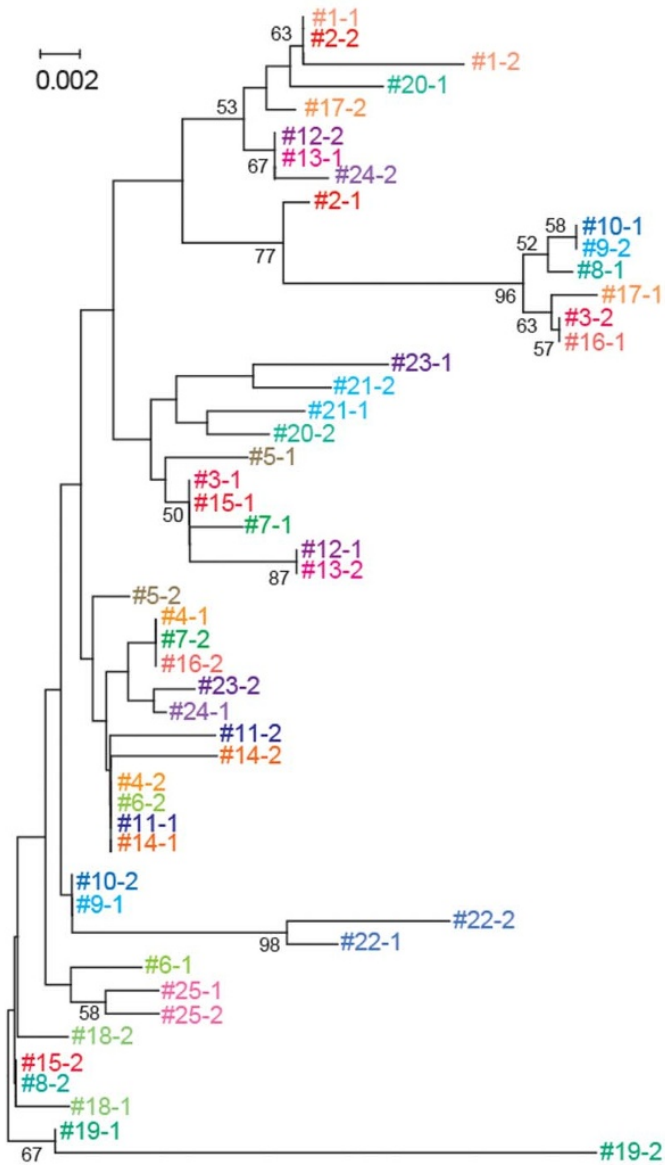

B

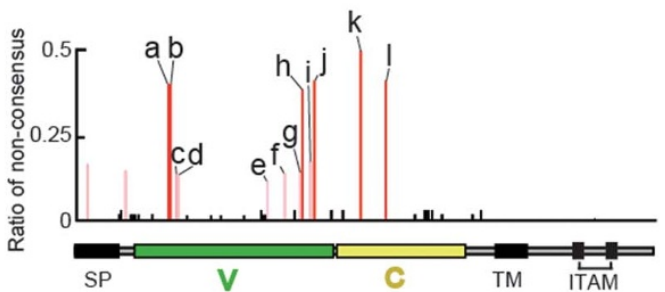

C

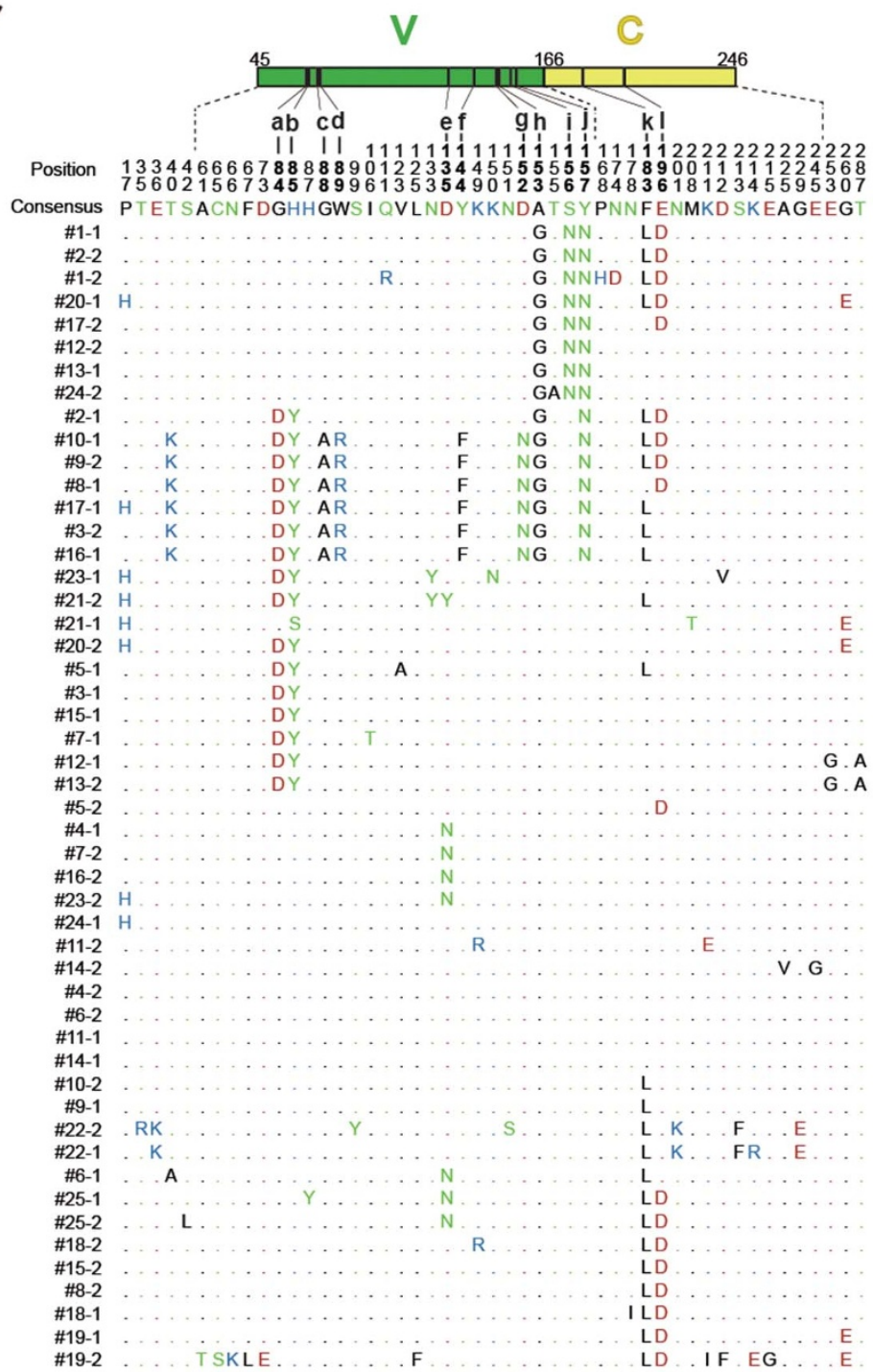

Figure 2 Polymorphisms of the allogenic leukocyte antigen, ALA. (A) A phylogenetic tree of 50 different NICIR3/ALA alleles identified in 25 different individuals (\#1-\#25). The tree was constructed with the unrooted Neighbor-Joining method. Bootstrap support values over $50 \%$ are shown at branches. Amino acid sequences were compared using the Clustal W program. (B) The extracellular domains of NICIR3/ALA consist of two Ig domains, V (48$165 \mathrm{aa}$ ) and C (166-247 aa). The variability of amino acid (aa) residues (a-1) in 50 NICIR3/ALA sequences from 25 animals are shown. The ratio of atypical aa residues was calculated for each aa position as follows: the number of samples with atypical aa residues was divided by total number of samples. Vertical bars represent variable aa residues: red, $>0.25$; pink, 0.04-0.25; and black, $<0.04$. Variable aa residues $(>0.04)$ are indicated by alphabets, a- 1 . The schematic diagram of NICIR3/ALA is shown on the bottom. SP, signal peptide; TM, transmembrane domain; ITAM, immunoreceptor tyrosinebased activation motif. The $\mathrm{V}$ and $\mathrm{C}$ domains are in green and yellow, respectively. (C) Polymorphic aa residues in the NICIR3/ALA sequences. Variable aa residues (a-1) in Fig. 2B are shown on the bottom. Hydrophobic residues are shown in black color, hydrophilic residues in green, acidic residues in red and basic residues in blue. GenBank accessions of the NICIR3/ALA alleles are AB723629-AB723678.

observed between the two $(\mathrm{R}=0.69$; Spearman's correlation coefficient, $\mathrm{p}<0.0001)$. These results indicate that the VLR-Bs of more distantly related haplotypes tend to possess higher reactivities to buffy coat leukocytes (supplementary Fig. 3). Therefore, we hereinafter refer to NICIR3 as the allogenic leukocyte antigen (ALA) in hagfish. To determine whether the allogenic NICIR3/ALA is directly recognized by VLR-Bs, an NICIR3/ALA was expressed in HEK293T cells and the interaction with allogenic VLR-Bs was examined using flow cytometry. Fig. 3D demonstrates the allogenic interaction between the NICIR3/ALA and the serum VLR-Bs.

NICIR3/ALA is expressed in phagocytic cells. To study the localization of NICIR3/ALA, we generated anti-NICIR3/ALA polyclonal antibodies in mice (supplementary Fig. 2A-D). In jawless fish, blood cells can be sorted into at least three different subpopulations by flow cytometry: erythrocytes, lymphocytes, and myeloid cells (Fig. 4A) 9,33,34. By staining the blood cells with antiNICIR3/ALA antibodies, we found that leukocytes (i.e. lymphocytes and myeloid cells) expressed NICIR3/ALA, whereas erythrocytes did not (Fig. 4B) and that VLR-B positive lymphocytes expressed NICIR3/ALA more than VLR-A positive lymphocytes (Fig. 4C). NICIR3/ALA signals were observed not only at the cell surface but also in the cytoplasm. To examine the subcellular localization of NICIR3/ALA, we first labeled the phagocytic leukocytes with an exogenous antigen, keyhole limpet hemocyanin (KLH), by intraperitoneal injection. Within 24 hours, approximately $10 \%$ of buffy coat 


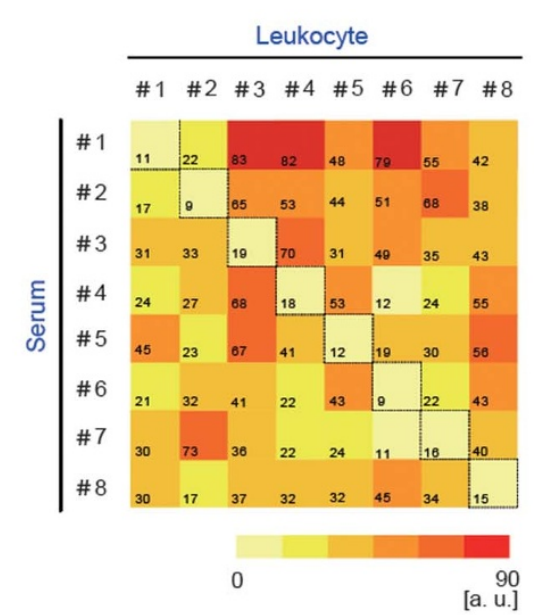

C Antigenicity scores and VLR-B binding

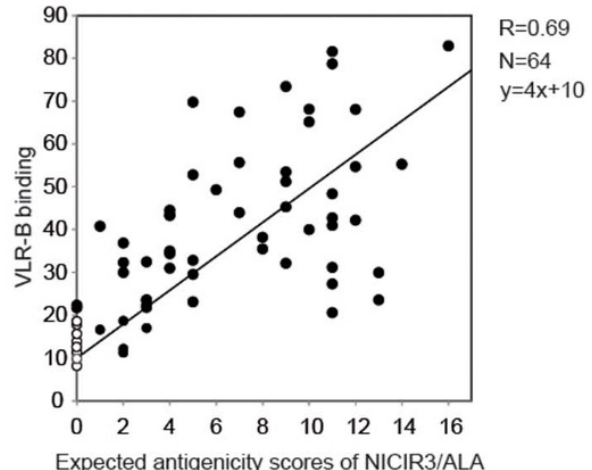

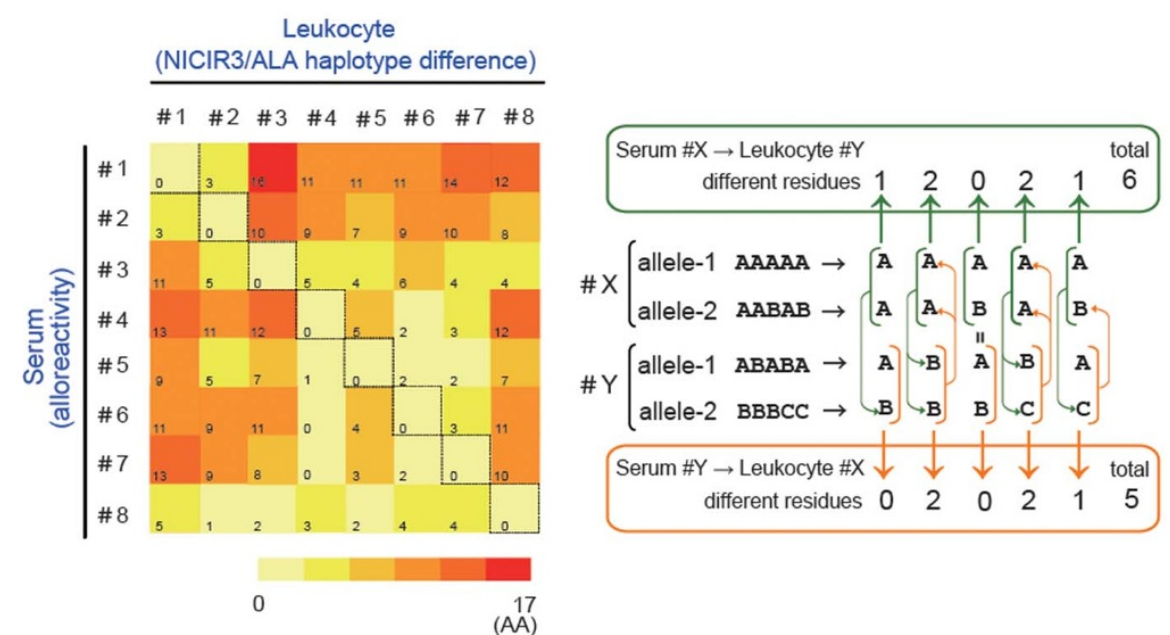

D

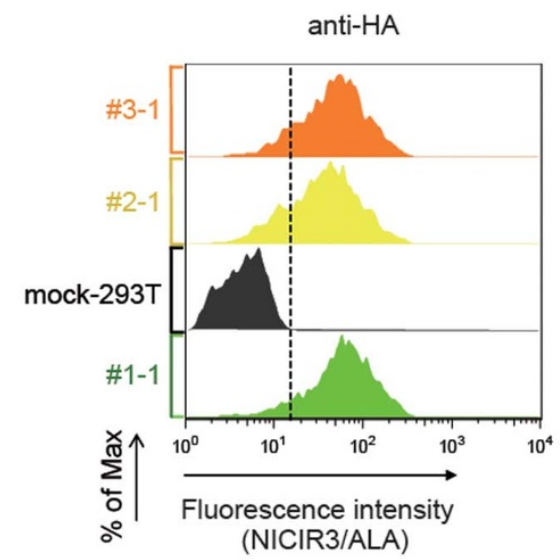

Figure 3 NICIR3/ALA haplotypes and allogenic reactivities. (A) Quantitative analysis of binding between VLR-B samples and buffy coat leukocytes. The buffy coat leukocytes and sera were isolated from eight different individuals (\#1-\#8). Serological tests shown in Fig. $1 \mathrm{D}$ were performed for all $8 \times 8$ combinations. The resulting signal intensities are shown as a heat map. (B) Haplotypes and expected antigenicities of NICIR3/ALA. Expected antigenicity scores were calculated for each pair of serum and buffy coat leukocyte for both alleles. A correlation between the alloreactivities (serum) and the amino acid residue differences (leukocyte) is shown as a heat map for all $8 \times 8$ combinations. The calculation of the number of residue differences is schematically shown on the right, where arrows indicate a given subtype of VLR-B reacting with a non-self-NICIR3/ALA. Antigenisity scores were calculated for both allele 1 and 2 by counting how many allogenic residues are contained in NICIR3/ALA without considering positions and species of aa residues. We also assumed that two alleles of NICIR3/ALA equally and independently contribute to the antigenicity. (C) Correlation between the antigenicity scores of NICIR3/ALA and VLR-B binding for alloreactivities. The allogenic antigenicity of NICIR3/ALA and binding score of VLR-B are shown in closed circles $(\mathbf{O})$. Self-self reactivities are shown in open circles $(\bigcirc)$. A positive correlation $(\mathrm{R}=0.69$; Spearman's correlation coefficient $)$ was observed between the expected antigenicity scores for NICIR3/ALA and VLR-B binding scores. (D) Alloreactivities of VLR-Bs to hemagglutinin (HA)tagged NICIR3/ALA molecules expressed in HEK293T cells (left). Three different haplotypes from three different animals, \#1, \#2 and \#3, were analyzed. Samples treated with the serum from animal \#1 were applied (right), and the binding of VLR-Bs was measured by flow cytometry. The mock-transfected HEK293T sample (mock-293T) was used as a negative control. The \#1 serum reacted with the \#2 allele-1 and \#3 allele-1 (allogenic) but not with the \#1 allele-1 NICIR3/ALA (self). Allele numbers, \#1-\#8, are the same in Fig. 2.

leukocytes were stained for KLH, and immunoelectron microscopy analyses demonstrated that most of these KLH-positive phagocytic cells were monocytes and granule like cells (supplementary Fig. 4A). Immunocytochemistry with the anti-NICIR3/ALA antibodies then demonstrated that NICIR3/ALA colocalized with phagocytosed KLH proteins in the phagocytic cells (Fig. 4D and supplementary Fig. 5). The colocalization of NICIR3/ALA and phagocytosed proteins were observed within a lysosomal compartment by colloidal gold immunostaining (Fig. 4E and supplementary Fig. 4B).

\section{Discussion}

In the present study, we found that hagfish VLR-Bs recognize an allogenic leukocyte antigen, ALA (previously reported as NICIR3). NICIR3/ALA is a transmembrane protein consisting of two domains, $\operatorname{IgV}$ and $\mathrm{IgC}$, in the extracellular region and two immuno-receptor tyrosine based activation motifs (ITAM) ${ }^{35}$ in the intracellular region (supplementary Fig. 2). We assume that NICIR3/ ALA transduces ligand signals to induce a set of genes for lymphocyte activation via ITAMs. From serological tests, NICIR3/ALA was 
A

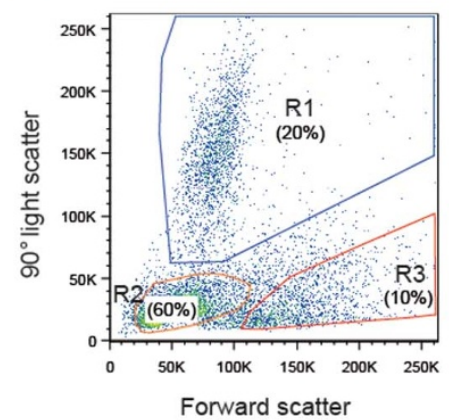

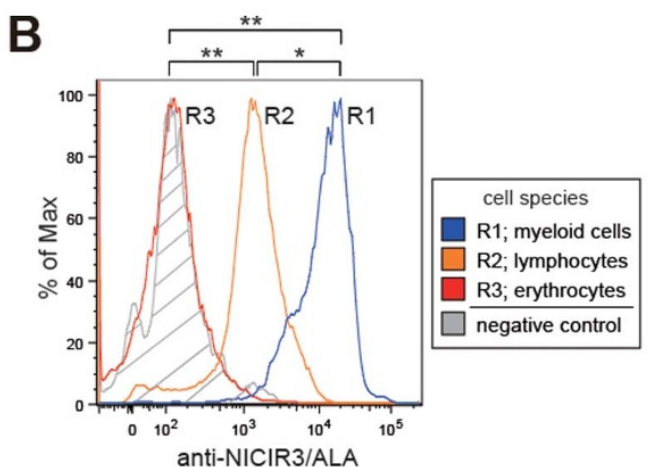
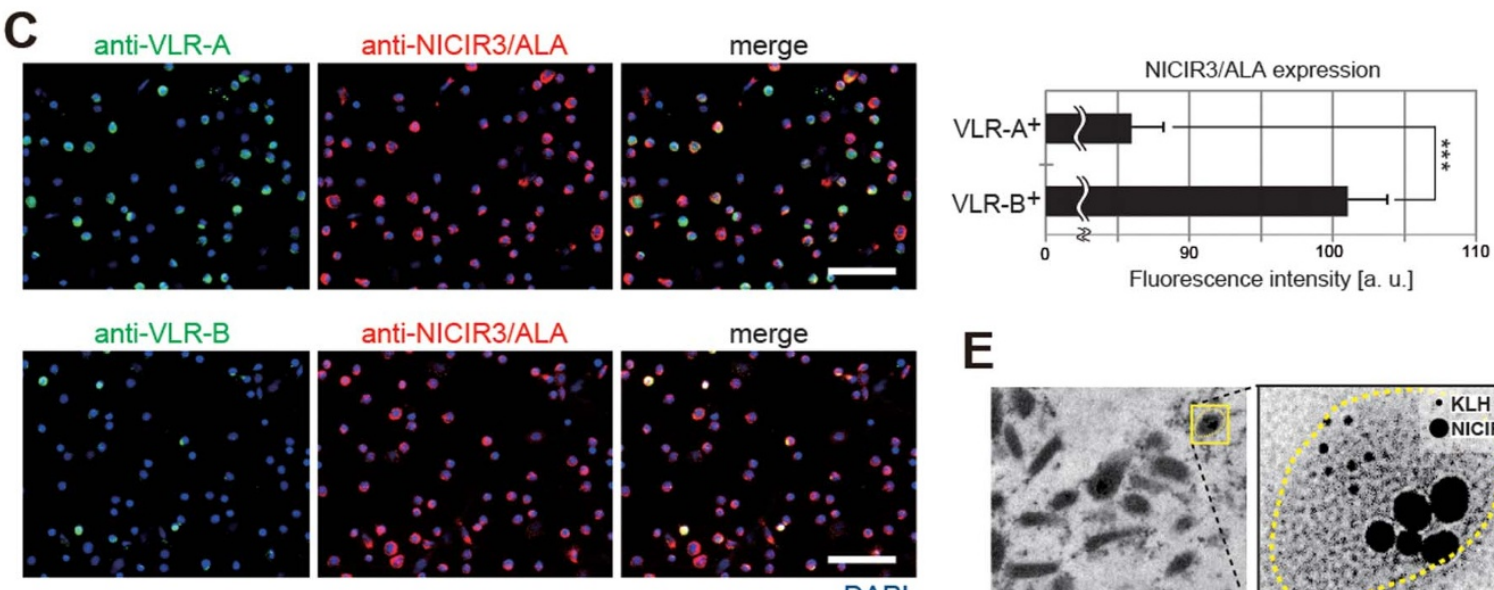

DAPI

E
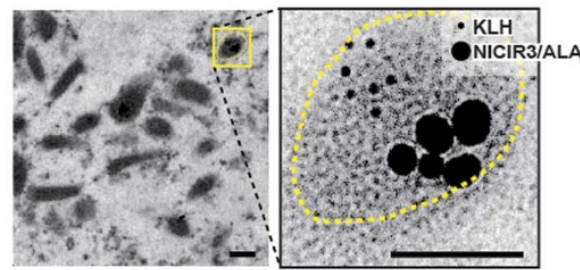

D
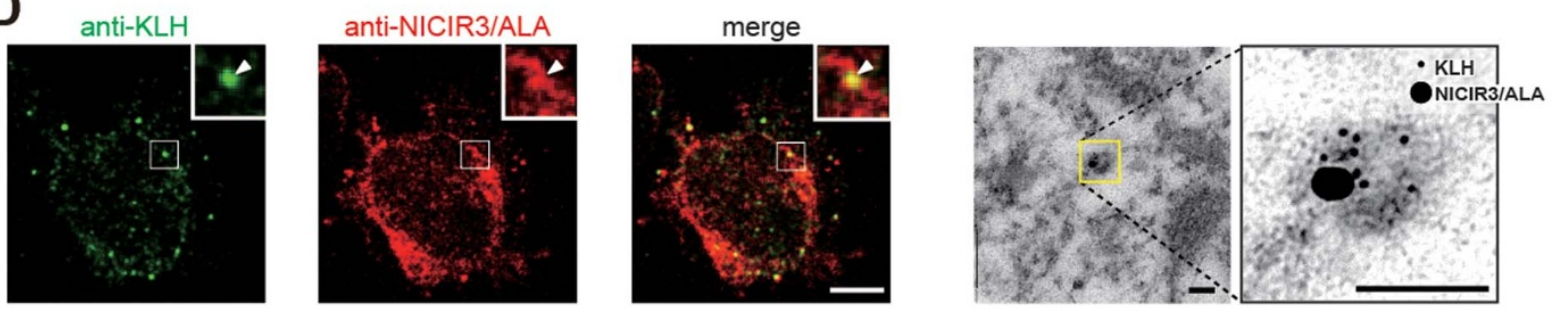

Figure $4 \mid$ Cellular and subcellular localization of NICIR3/ALA. (A) Flow cytometric fractionation of peripheral blood cells. Forward vs. $90^{\circ}$ light scattering profiles are shown. Giemsa staining of the fractionated cells is shown on the right. Fractions R1, R2, and R3 contained myeloid cells, lymphocytes and erythrocytes, respectively. Scale bars indicate $10 \mu \mathrm{m}$. (B) Flow cytometric analysis of NICIR3/ALA expression in fractions, R1-R3. NICIR3/ALA is detected in R1 (myeloid cells) and R2 (lymphocytes), but not in R3 (erythrocytes). Cells stained with pre-immune serum was used as a negative control; $\mathrm{n}=8$; * $P<0.05$ and ${ }^{* *}, P<0.01$ from Welch's t-test. (C) Immunostaining of buffy coat leukocytes with antibodies against VLR-A or VLR-B (green) and NICIR3/ALA (red). NICIR3/ALA was expressed in VLR-B positive cells more than in VLR-A cells. $\mathrm{n}=4$; ***, $P<0.001$ from Welch's t-test. Scale bars indicate $50 \mu \mathrm{m}$. (D) Confocal microscopy analysis of NICIR3/ALA -positive cells. One day after the injection of KLH, buffy coat leukocytes were stained with anti-NICIR3/ALA and anti-KLH antibodies. KLH (green) colocalized with the NICIR3/ALA staining (red) in leukocytes. $\mathrm{n}=3$. A scale bar is $2 \mu \mathrm{m}$. (E) Colocalization of KLH and NICIR3/ALA in a phagocytic leukocyte by immunoelectron microscopy. As in (D), buffy coat leukocytes were stained with anti-NICIR3/ALA and anti-KLH antibodies; $\mathrm{n}=3$. Two representative data are shown. NICIR3/ALA and KLH were labeled with $30 \mathrm{~nm}$ and $6 \mathrm{~nm}$ gold particles, respectively. Enlarged views are shown on the right. Scale bars indicate $100 \mathrm{~nm}$.

shown to be a major allogenic antigen for the hagfish VLR-Bs (Fig. 3 and supplementary Fig. 3). Because the $V L R-B$ genes are randomly assembled in an antigen-independent manner ${ }^{16}$, self-reactive VLRBs must be generated in the hagfish. However, no VLR-Bs were found to react to autologous antigens. Thus, it is conceivable that the VLR-B repertoire is negatively selected for self antigens including NICIR3/ALA. Flow cytometric analysis showed that NICIR3/ALA is expressed in myeloid cells and lymphocytes (Fig. 4B). We also found that NICIR3/ALA colocalized with that of phagocytosed proteins within leukocytes (Fig. 4D and E and supplementary Fig. 4B). Furthermore, using the proximity ligation assay, we demonstrated close association of NICIR3/ALA and phagocytosed protein antigens both in the cytoplasm and on the cell surface (supplementary Fig. 7 and 8). Because NICIR3/ALA-protein antigen association requires both the polymorphic $\mathrm{V}$ and $\mathrm{C}$ domains, these domains may together constitute a ligand-binding site for various protein antigens (supplementary Fig. 8).

It was reported that VLR-A cells might be selected in the gill, a thymus like tissue ${ }^{36}$. A search for the thymus in hagfish was unsuccessful, most likely because the thymus like tissue is lost in the adult. We also analyzed the hematopoietic nest ${ }^{37}$ a candidate lymphoid organ, located around intestine where expression of VLR-A and VLR-B was prominent (supplementary Fig. 10A-C). We examined whether NICIR3/ALA is expressed in the hematopoietic nest, and found that NICIR3/ALA positive cells are located in close proximity to VLR-positive cells (supplementary Fig. 10D and E). It is tempting 
to assume that NICIR3/ALA is involved in the elimination of selfreactive VLR cell. In the hagfish, it has been assumed that VLR-As function like mammalian TCRs ${ }^{38}$. It is interesting to study whether NICIR3/ALA is also involved in the VLR-A selection.

The evolutionary origin of adaptive immunity represents one of the greatest biological enigmas. An obvious advantage of adaptive immunity is the capacity to cope with novel pathogens using a wide variety of antigen receptors and it is widely held that this emerged in vertebrates during the Cambrian explosion approximately 500 million years ago ${ }^{8,10,39}$. For diversification of antigen receptor genes, jawless vertebrates employ a copy-choice mechanism ${ }^{16}$, whereas jawed vertebrates utilize $\mathrm{V}(\mathrm{D}) \mathrm{J}$ recombination, a reverse reaction involving the accidental insertion of transposons that occurred during their evolution ${ }^{18}$. Although both systems diversify antigen receptor genes by entirely different mechanisms, the random generation of a large variety of antigen receptors potentially produces hazardous self-reactive repertoire in both systems. Through this study, we demonstrated that VLR-B molecules in jawless fish undergo negative selection to establish self-tolerance. It is quite interesting that both jawless and jawed vertebrates utilize negative selection to control the repertoire of their antigen receptors despite both systems having evolved these antigen receptors from unrelated molecular pathways. Our findings provide important insights into the evolution of immune systems and the immunological tolerance in adaptive immunity in the context of the clonal selection theory proposed by Burnet in $1957^{21}$.

\section{Methods}

Hagfish samples. Adult hagfish (Eptatretus burgeri) were captured in the Pacific Ocean off the coast of Misaki, Kanagawa, Japan and were cultivated at $15^{\circ} \mathrm{C}$ in artificial sea water (Marine Art HG, Kotobuki). The hagfish were anesthetized in $500 \mathrm{mg} / \mathrm{L}$ of MS222 (ethyl $m$-aminobenzoate methanesulfonate; Nacalai Tesque). Peripheral blood samples were collected from the caudal subcutaneous sinus and placed in hagfish phosphate-buffered saline (hagfish PBS) containing $30 \mathrm{mM}$ EDTA $^{27}$. Leukocytes were prepared from a buffy coat fraction as previously described $^{27,28}$. The serum was prepared from peripheral blood clotted at $4^{\circ} \mathrm{C}$ overnight. To label the phagocytic cells, $100 \mu \mathrm{g}$ of keyhole limpet hemocyanin (KLH) (Sigma) and $100 \mu \mathrm{l}$ of Freund's complete adjuvant (Thermo) were intraperitoneally injected into the hagfish, and buffy coat leukocytes were collected one day after. All animal experiments were approved by the Animal Care Committee in the University of Tokyo.

Generation of antibodies against VLRs and NICIR3/ALA. Two monoclonal antibodies were produced in mice for VLR-A and VLR-B using synthetic peptides conjugated with bovine serum albumin (BSA). Peptides used are listed in supplementary Table 1. Lymph node cells were collected and fused with Ig-negative myeloma cells. Hybridoma clones producing antibodies against VLR-A (2A-1G; IgG2b, $\kappa)$ and VLR-B (25D-9C; IgG1, $\kappa)$ were identified by ELISA, immunoblotting and immunocytochemistry. Anti-VLR-A and anti-VLR-B polyclonal antisera were produced by immunizing rabbits with BSA-conjugated synthetic peptides listed in Supplementary Table 1. Anti- NICIR3/ALA polyclonal antisera were produced in mice. Antibody reactivities were examined for 16 different haplotype NICIR3s/ALAs expressed in HEK293T. All antibodies were affinity-purified with peptide antigens.

Enzyme-linked immunosorbent assay (ELISA). Diluted hagfish sera and VLR-A or VLR-B peptides (50 ng/well) in $20 \mathrm{mM}$ phosphate saline were loaded on 96 -well plates (Nunc) pretreated with $1 \%(\mathrm{wt} / \mathrm{v})$ BSA in PBS for $1 \mathrm{hr}$. Diluted samples were incubated for $2 \mathrm{hrs}$ at $37^{\circ} \mathrm{C}$. VLRs were detected with anti-VLR-A (2A-1G) or antiVLR-B (25D-9C) monoclonal antibodies and then with HRP-conjugated goat antimouse IgG $(1: 2,000$, Invitrogen). ELISA signals were detected with a chromogenic peroxidase substrate, 3,3',5,5'-tetramethylbenzidine (TMB) (Pierce). Absorbance at $450 \mathrm{~nm}$ was quantified with BertholdTech TriStar LB941multimode plate reader.

Serological tests for buffy coat leukocytes and serum. Buffy coat leukocytes were placed on glass slides $\left(2 \times 10^{5}\right.$ cells per slide), treated with $4 \%(\mathrm{wt} / \mathrm{v})$ paraformaldehyde in hagfish PBS for $5 \mathrm{~min}$, and subsequently fixed with zinc formalin (Polysciences, Inc.) in PBS for $20 \mathrm{~min}$. The slides were rinsed in TBS ( $50 \mathrm{mM}$ Tris and $150 \mathrm{mM} \mathrm{NaCl}, \mathrm{pH} 7.6)$ three times. Samples were blocked with $5 \%(\mathrm{w} / \mathrm{v})$ skim milk in TBS and incubated with inactivated hagfish serum $(20 \%)$ in TBS overnight at room temperature. The slides were washed three times and treated with primary antibodies (anti-VLR-B, 25D-9C) for $1 \mathrm{hr}$ at room temperature. Signals were visualized with secondary antibodies conjugated with Alexa Fluor 555 (1:400, Molecular Probes). Nuclear counterstaining was performed with DAPI (Roche). The stained cells were analyzed with an Olympus Model IX70 fluorescence microscope using a cooled CCD camera (model ORCA-R2, Hamamatsu Photonics). Region of interest (ROI) was selected by DAPI. Fluorescent intensity (VLR-B binding) per leukocyte was measured in ROI using ImageJ software (version 1.46, http:// rsbweb.nih.gov/ij/) ( $\mathrm{n}>$ randomly chosen 300 cells/sample). Mean of fluorescence intensities scores are shown in boxes (Fig. 3A).

Interactions of serum VLR-Bs to NICIR3/ALA expressed in HEK293T cells. The NICIR3/ALA coding sequence was amplified by PCR and fused to the Igא signal peptide and hemagglutinin (HA) epitope. NICIR3/ALA-L primer: SfiI5' - TTGGCCCAGCCGGCCCCGCACGCCATATGTGTT - $3^{\prime}$. NICIR3/ALA-R Primer: PstI- 5' - TTCTGCAGTCATGCTGTGTGGAGTGTTTC - $3^{\prime}$. NICIR3/ALA expression vector was transfected into HEK-293T cells using Lipofectamine 2000 (Invitrogen). The cells were cultured in DMEM supplemented with $10 \%$ (v/v) FBS. To avoid nonspecific binding, the serum was pre-incubated overnight with HEK293T cells transfected with a mock vector (pDisplay). HEK293T cells expressing NICIR3/ ALA were treated with $10 \%(\mathrm{v} / \mathrm{v})$ pre-absorbed serum for $1 \mathrm{hr}$, anti-VLR-B monoclonal antibody (25D-9C) for $1 \mathrm{hr}$, and Alexa Fluor 488-conjugated goat antimouse IgG $(1: 400$, Molecular Probes $)$ at $37^{\circ} \mathrm{C}$. HA-tagged proteins were stained with Alexa488-conjugated anti-HA monoclonal antibody (1:200, Molecular Probes) for flow cytometric analysis (BD FACS Caliber).

Microarray analysis. A custom microarray $(4 \times 44 \mathrm{~K}$ probes, Agilent) was made, using approximately 24,000 EST sequences from hagfish leukocytes ${ }^{32}$. Total RNA was purified from leukocytes or erythrocytes of the hagfish buffy coat $(n=4)$. Cy3- or Cy5-labeled cRNA probes were prepared with Agilent Low-RNA Input Linear Amplification Kit (Agilent) and hybridized to the custom microarray according to the manufacturer's instruction (Agilent). The hybridized arrays were scanned and quantified by Agilent DNA microarray scanner and Feature Extraction (Agilent). Data analysis was performed with Microsoft Excel.

RT-PCR and sequence analysis. NICIR3/ALA genes were amplified by PCR from cDNA prepared from buffy coat leukocytes under the following condition: $94^{\circ} \mathrm{C}$ for $2 \mathrm{~min}$ followed by $35 \mathrm{cycles}$ of $94^{\circ} \mathrm{C}$ for $30 \mathrm{~s}, 60^{\circ} \mathrm{C}$ for $30 \mathrm{~s}$, and $72^{\circ} \mathrm{C}$ for $1 \mathrm{~min}$, with a final hold at $72^{\circ} \mathrm{C}$ for $5 \mathrm{~min}$. NICIR3/ALA primers used in this study are listed in Table S2. Cloning and sequence analysis of the genes were performed as described previously ${ }^{16}$. Ten clones were sequenced for each allele. Genbank accession numbers are AB723629-AB723678.

Phylogenetic tree ofNICIR3/ALA-related proteins. Amino acid sequences were compared using the Clustal X program. A phylogenetic tree was made using the Neighbor-Joining (NJ) method. Evolutionary distances were calculated with the JTT matrix-based method. Both NJ analysis and bootstrap analysis were carried out with MEGA 5.05.

Sorting of hagfish blood cells. Hagfish blood cells were sorted into three discrete subpopulations (lymphocytes, myeloid cells and erythrocytes) based on the forward vs. $90^{\circ}$ light-scattering profiles from flow cytometry ${ }^{9,33,34}$. Cells were collected from each subpopulation using FACS Aria (BD).

Flow cytometric analysis. Buffy coat leukocytes (and erythrocytes, representing 10\% of the cells) from peripheral blood samples were fixed with hagfish PBS containing $10 \%$ formaldehyde for $5 \mathrm{~min}$. Samples were washed twice and stained with mouse anti- NICIR3/ALA polyclonal antibodies for $1 \mathrm{hr}$ on ice. The samples were then washed twice and stained with fluorescent-conjugated secondary antibodies, Alexa Fluor 488-conjugated goat anti-mouse IgG (1:400, Molecular Probes). Data were obtained using FACS Aria (BD) and analyzed with FlowJo software (Treestar). For staining and washing, PBS with 0.5\% BSA and 2 mM EDTA ( $\mathrm{pH} 7.4$ ) was used. Unimmunized mouse serum was used as a negative control for primary antibodies.

Confocal imaging of phagocytosed proteins. To label the phagocytic cells, $100 \mu \mathrm{g}$ of proteins (KLH or OVA) and $100 \mu \mathrm{l}$ of Freund's complete adjuvant (Thermo) were intraperitoneally injected into the hagfish. Buffy coat leukocytes were collected one day after. The cells were fixed with 4\% PFA for $1 \mathrm{hr}$ and incubated at room temperature for 2 hrs with rabbit anti-KLH antibodies (Rockland) or anti-OVA antibodies (Delta Biolabs), and with mouse anti- NICIR3/ALA antibodies. Samples were then stained with goat anti-rabbit IgG Alexa Fluor 488 (1:300, Molecular Probes) and goat anti-mouse IgG, Alexa Fluor 555 ( $1: 300$, Molecular Probes). After washing with $0.01 \%$ Triton-X100-PBS, confocal images were obtained with an upright confocal microscope, Olympus Model FV500.

Immunoelectron microscopy of phagocytosed proteins. To label the phagocytic cells, $100 \mu \mathrm{g}$ of KLH proteins and $100 \mu \mathrm{l}$ of Freund's complete adjuvant (Thermo) were intraperitoneally injected into the hagfish. Buffy coat leukocytes were collected one day after. The cells were fixed with 4\% PFA for $1 \mathrm{hr}$ and embedded in LR-white resin after dehydration with ethanol. Samples were sliced in 60-70 $\mathrm{nm}$ sections, incubated with rabbit anti-KLH antibodies (Rockland) for 2 hrs at room temperature, and then with goat anti-rabbit IgG labeled with $6 \mathrm{~nm}$ colloidal gold ( 1 : 30, Jackson Immuno Research Laboratories, Inc.) for $1 \mathrm{hr}$. After KLH staining, samples were incubated with anti- NICIR3/ALA antibodies and then with goat anti mouse IgG labeled with $30 \mathrm{~nm}$ colloidal gold (1:30, EY Laboratories, Inc.). After incubation with secondary antibodies, the samples were postfixed for $10 \mathrm{~min}$ in $2 \%$ $(\mathrm{wt} / \mathrm{v})$ glutaraldehyde and $1 \%(\mathrm{wt} / \mathrm{v})$ osmium tetroxide. After washing with PBS, 
samples were stained with saturated aqueous solution of uranyl acetate and lead citrate, and analyzed by electron microscopy (JEOL, JEM-1400).

Immunocytochemistry. For immunocytochemistry, buffy coat leukocyte samples were fixed in zinc formalin fixative (Polysciences, Inc.). Samples were treated with $10 \%(\mathrm{v} / \mathrm{v})$ goat serum for $1 \mathrm{hr}$ after antigen retrieval by heating at $121^{\circ} \mathrm{C}$ for $20 \mathrm{~min}$ at $100 \mathrm{kPa}$ in $0.01 \mathrm{M}$ citric acid, $\mathrm{pH}$ 6. The samples were incubated with primary antibodies for $7 \mathrm{hrs}$ at $4{ }^{\circ} \mathrm{C}$ and then with secondary antibodies. Nuclear counterstaining was performed with DAPI (Roche). The stained cells were analyzed with Olympus Model IX70 fluorescence microscope with a cooled CCD camera (model ORCA-R2, Hamamatsu Photonics). Mean fluorescence intensities of NICIR3/ALA were measured in VLR-A and VLR-B positive cells selected by Image software (n > 300 VLR-positive cells/sample).

1. Finstad, J. \& Good, R. A. The evolution of the immune response. III. Immunologic responses in the lamprey. J Exp Med 120, 1151-1168 (1964).

2. Hildemann, W. H. \& Thoenes, G. H. Immunological responses of Pacific hagfish. I. Skin transplantation immunity. Transplantation 7, 506-521 (1969).

3. Linthicum, D. S. \& Hildemann, W. H. Immunologic responses of Pacific hagfish. III. Serum antibodies to cellular antigens. J Immunol 105, 912-918 (1970).

4. Pollara, B., Litman, G. W., Finstad, J., Howell, J. \& Good, R. A. The evolution of the immune response. VII. Antibody to human "O" cells and properties of the immunoglobulin in lamprey. J Immunol 105, 738-745 (1970).

5. Good, R. A., Finstad, J. \& Litman, G. W. in The Biology of Lampreys II: Immunology, Hardisty, M. V., Potter, I. C., Eds. (Academic, London, 1972), 405-432 (1972).

6. Burnet, F. M. "Self-recognition" in Colonial Marine Forms and Flowering Plants in relation to the Evolution of Immunity. Nature 232, 230-235 (1971).

7. Raison, R. L., Gilbertson, P. \& Wotherspoon, J. Cellular requirements for mixed leucocyte reactivity in the cyclostome, Eptatretus stoutii. Immunol Cell Biol 65 (Pt2), 183-188 (1987)

8. Flajnik, M. F. Comparative analyses of immunoglobulin genes: surprises and portents. Nat Rev Immunol 2, 688-698 (2002).

9. Pancer, Z. et al. Somatic diversification of variable lymphocyte receptors in the agnathan lamprey. Nature 430, 174-180 (2004)

10. Cooper, M. D. \& Alder, M. N. The Evolution of Adaptive Immune Systems. Cell 124, 815-822 (2006)

11. Medzhitov, R. \& Janeway, C. A. Jr. Decoding the patterns of self and non-self by the innate immune system. Science 296, 298-300 (2002).

12. Akira, S., Uematsu, S. \& Takeuchi, O. Pathogen recognition and innate immunity. Cell 124, 783-801 (2006).

13. Alder, M. N. et al. Diversity and function of adaptive immune receptors in a jawless vertebrate. Science 310, 1970-1973 (2005).

14. Han, B. W., Herrin, B. R., Cooper, M. D. \& Wilson, I. A. Antigen recognition by variable lymphocyte receptors. Science 321, 1834-1837 (2008).

15. Alder, M. N. et al. Antibody responses of variable lymphocyte receptors in the lamprey. Nat Immunol 9, 319-327 (2008).

16. Nagawa, F. et al. Antigen receptor genes of the agnathan lamprey are assembled by a process involving copy choice. Nat Immunol 8, 206-213 (2007).

17. Rogozin, I. B. et al. Evolution and diversification of lamprey antigen receptors: evidence for involvement of an AID-APOBEC family cytosine deaminase. Nat Immunol 8, 647-656 (2007).

18. Sakano, H., Huppi, K., Heinrich, G. \& Tonegawa, S. Sequences at the somatic recombination sites of immunoglobulin light-chain genes. Nature 280, 288-294 (1979).

19. Agrawal, A., Eastman, Q. M. \& Schatz, D. G. Transposition mediated by RAG and RAG2 and its implications for the evolution of the immune system. Nature 394, 744-751 (1998).

20. Hiom, K., Melek, M. \& Gellert, M. DNA transposition by the RAG1 and RAG2 proteins: a possible source of oncogenic translocations. Cell 94, 463-470 (1998).

21. Burnet, F. M. Clonal Selection Theory: A modification of Jerne's theory of antibody production using the concept of clonal selection. Aust J Sci 20, 67-69 (1957)

22. Rajewsky, K. Clonal selection and learning in the antibody system. Nature $\mathbf{3 8 1}$, 751-758 (1996).

23. Goodnow, C. C., Sprent, J., Fazekas de St Groth, B. \& Vinuesa, C. G. Cellular and genetic mechanisms of self tolerance and autoimmunity. Nature 435, 590-597 (2005)
24. Sprent, J. \& Cho, J. H. Self/non-self discrimination and the problem of keeping T cells alive. Immunol Cell Biol 86, 54-56 (2008).

25. Guo, P. et al. Dual nature of the adaptive immune system in lampreys. Nature 459, 796-801 (2009).

26. Kasamatsu, J. et al. Identification of a third variable lymphocyte receptor in the lamprey. Proc Natl Acad Sci USA 107, 14304-14308 (2010).

27. Pancer, Z. et al. Variable lymphocyte receptors in hagfish. Proc Natl Acad Sci USA 102, 9224-9229 (2005).

28. Kishishita, N. et al. Regulation of antigen-receptor gene assembly in hagfish. EMBO Rep 11, 126-132 (2010)

29. Landsteiner, K. Uber Agglutinationserscheinungen normal menschlichen Blutes. Wiener Klinische Wochenschrift 14, 1132-1134 (1901).

30. Ravindranath, M. H., Kaneku, H., El-Awar, N., Morales-Buenrostro, L. E. \& Terasaki, P. I. Antibodies to HLA-E in nonalloimmunized males: pattern of HLA-Ia reactivity of anti-HLA-E-positive sera. J Immunol 185, 1935-1948 (2010).

31. Suzuki, T., Shin-I, T., Kohara, Y. \& Kasahara, M. Transcriptome analysis of hagfish leukocytes: a framework for understanding the immune system of jawless fishes. Dev Comp Immunol 28, 993-1003 (2004).

32. Haruta, C., Suzuki, T. \& Kasahara, M. Variable domains in hagfish: NICIR is a polymorphic multigene family expressed preferentially in leukocytes and is related to lamprey TCR-like. Immunogenetics 58, 216-225 (2006).

33. Gilbertson, P., Wotherspoon, J. \& Raison, R. L. Evolutionary development of lymphocyte heterogeneity: leucocyte subpopulations in the Pacific hagfish. Dev Comp Immunol 10, 1-10 (1986).

34. Newton, R. A., Raftos, D. A., Raison, R. L. \& Geczy, C. L. Chemotactic responses of hagfish (Vertebrata, Agnatha) leucocytes. Dev Comp Immunol 18, 295-303 (1994).

35. Isakov, N. Immunoreceptor tyrosine-based activation motif (ITAM), a unique module linking antigen and $\mathrm{Fc}$ receptors to their signaling cascades. J Leukoc Biol 61 (1), 6-16 (1997)

36. Bajoghli, B. et al. A thymus candidate in lampreys. Nature 470, 90-94 (2010).

37. Tanaka, Y., Saito, Y. \& Gotoh, H. Vascular architecture and intestina hematopoietic nests of two cyclostomes, Eptatretus burgeri and ammoncoetes of Entosphenus reissneri: a comparative morphological study. J Morphol 170, 71-93 (1981).

38. Boehm, T. et al. VLR-based adaptive immunity. Annu Rev Immunol 30, 203-220 (2012).

39. Litman, G. W., Cannon, J. P. \& Dishaw, L. J. Reconstructing immune phylogeny: new perspectives. Nat Rev Immunol 5, 866-879 (2005).

\section{Acknowledgements}

This work was supported by grants in aid from the Ministry of Education, Culture, Sports, Science and Technology in Japan. T.I. was supported by the JST PRESTO program. H.N. and H.S. were supported by grants from the Japan Foundation for Applied Enzymology. We thank Drs. M.F. Flajnik, K. Ishiguro, and members of our laboratory for valuable suggestions and discussions.

\section{Author contributions}

H.T. designed research; H.T., T.I., S.M. and Y.M. performed research; H.T., A.M., N.I., H.N. and H.S. analyzed data; and H.T., H.N. and H.S. wrote the paper.

\section{Additional information}

Supplementary information accompanies this paper at http://www.nature.com/ scientificreports

Competing financial interests: The authors declare no competing financial interests License: This work is licensed under a Creative Commons Attribution-NonCommercial-ShareAlike 3.0 Unported License. To view a copy of this license, visit http://creativecommons.org/licenses/by-nc-sa/3.0/

How to cite this article: Takaba, H. et al. A major allogenic leukocyte antigen in the agnathan hagfish. Sci. Rep. 3, 1716; DOI:10.1038/srep01716 (2013). 